\title{
Ahorro de Gas Natural en la Cocción de Pastas
}

Eduardo Juan Cavanagh ${ }^{1}$

\section{Resumen}

El agotamiento de recursos fósiles, de los cuales depende la sociedad moderna, ha llevado al hombre a la búsqueda de formas de reducir el uso de energía, sin afectar, en lo posible, su calidad de vida. Este artículo propone un nuevo método para cocinar pastas que reduciría el consumo de gas natural doméstico. El cálculo del ahorro de energía se basó en el consumo de pastas en Argentina y en el flujo estándar de gas en hornallas de hornos domésticos en Argentina. Se determinó que se podrían ahorrar 8,3 millones de Nm3 de gas, que representa un $0,086 \%$ del consumo de gas doméstico. Si bien este ahorro no es significativo en Argentina, esta propuesta invita al replanteo de costumbres afincadas en la sociedad que podrían modificarse en pos del ahorro energético, sin comprometer la calidad de vida.

Palabras Clave: Cocción de Pastas, Eficiencia Energética. 


\section{Abstract}

The depletion of fossil fuels that modern society depends on, has led man to search ways to reduce energy use without affecting life quality. This paper proposes a new pasta cooking method that reduces the consumption of domestic natural gas. Energy savings calculations were based on pasta consumption in Argentina and the standard flow gas of domestic furnace burners in Argentina. It was determined that 8.3 million $\mathrm{Nm} 3$ of gas, representing a $0.086 \%$ of domestic gas consumption, could be saved. While this savings is not significant in Argentina, this proposal invites to reconsider customs settled in the society that could be modified in pursuit of energy savings without compromising life quality.

Keywords: Cooking Pasta, Energy Efficiency. 


\section{Introducción}

Las instrucciones de cocción de los paquetes de pasta indican que hay que arrojar la pasta en agua hirviendo y dejarla cocinar por una cantidad definida de minutos que, dependiendo del tipo de pasta, se encuentra entre 4 y 10 minutos, siendo 7 minutos el promedio (ver Tabla 1). Sin embargo, con el ahorro de energía en mente, uno podría preguntarse: una vez que el agua junto con la pasta ha hervido, ¿es necesario mantener la hornalla prendida mientras se cocinan los fideos, o con el calor residual que permanece en el agua se pueden cocinar a fuego apagado? Es sabido que el agua posee una capacidad calorífica relativamente alta comparada con otras sustancias $\left(\mathrm{Cc}, \mathrm{H} 2 \mathrm{O} \approx 1 \mathrm{kcal} /{ }^{\circ} \mathrm{C} / \mathrm{kg}\right)$, lo cual indica que la temperatura del agua permanecerá caliente un tiempo considerable luego de haberse apagado el fuego. Sabido es también que la temperatura del agua nunca excede $100{ }^{\circ} \mathrm{C}$, independientemente de la intensidad del fuego. Por lo tanto, se postuló la hipótesis de que si una vez alcanzado el punto de ebullición se quita la fuente de calor (es decir, se apaga la hornalla), la temperatura del agua no variará considerablemente durante el tiempo estipulado de cocción, y la pasta se cocinará con normalidad.

Tabla 1. Tiempo de cocción de algunos tipos y marcas populares de pasta en Argentina

\begin{tabular}{lll}
\hline Tipo de Pasta & Marca & Tiempo de Cocción (minutos) \\
\hline Spaghetti & Lucchetti & 10 \\
& Matarazzo & 7.5 \\
& Don Vicente & 8 \\
\hline Tallarines & Lucchetti & 6 \\
& Don Vicente & 8 \\
\hline Fettuccini & Don Vicente & 8 \\
& Matarazzo & 6 \\
\hline Bucattini & Lucchetti & 6 \\
\hline Foratini & Matarazzo & 9 \\
\hline Mostachol & Lucchetti & 9 \\
\hline MostacholRayado & Matarazzo & 9 \\
\hline Tirabuzón & Lucchetti & 8 \\
& Matarazzo & 8 \\
\hline Dedalitos & Lucchetti & 5 \\
\hline Cabello de Angel & Matarazzo & 4 \\
\hline Ave María & Lucchetti & 5 \\
& Matarazzo & 5 \\
\hline
\end{tabular}




\begin{tabular}{lll}
\hline Moño & Lucchetti & 7 \\
& Matarazzo & 7 \\
\hline Coditos & Lucchetti & 8 \\
\hline & Matarazzo & 8 \\
\hline Municiones & Matarazzo & 5 \\
\hline Nidos Fettuccine & Lucchetti & 6 \\
\hline
\end{tabular}

Durante la cocción de las pastas, los gránulos de almidón contenidos en las mismas absorben agua y se hinchan irreversiblemente, produciéndose el proceso de gelatinización. La temperatura a la cual comienza a producirse la gelatinización se denomina "temperatura de gelatinización" o "pasting temperature", y suele encontrarse entre los 55 y $83^{\circ} \mathrm{C}$, dependiendo del tipo de almidón (KenjiLopez-Alt, 2010; Villagra, 2010; Vaclavik, 2002; Salinas Moreno, Herrera Corredor, Castillo Merino y Pérez Herrera, 2003; Ikegwu, Okechukwu y Ekumankana, 2010). Lo cual indica que la pasta se cocinaría incluso con un descenso significativo de la temperatura de ebullición del agua.

Esta investigación consiste de tres partes. En la primera se introducirá un nuevo método para cocinar pastas, el cual utiliza menor cantidad de gas que el método convencional y, por ende, ahorra energía. En la segunda parte se determinará el descenso de temperatura en una cacerola con agua luego de 10 minutos de haberse retirado la fuente de calor y siendo la temperatura inicial $100^{\circ} \mathrm{C}$. Se consideraron 10 minutos para verificar que este método funcionaría incluso con pastas que necesiten el mayor tiempo de cocción (ver Tabla 1). El objetivo de esta segunda parte es evaluar si, en este tiempo, la temperatura disminuye por debajo de la temperatura de gelatinización. En la tercera parte se calculará cuánta energía y gas se ahorraría si se utilizara el nuevo método de cocción de pastas en Argentina, con un tiempo promedio de cocción de 7 minutos.

\section{Primera parte: Nuevo método para cocinar pastas}

Para comprobar la hipótesis enunciada en la introducción, se realizó un experimento con pastas tipo penne, macarrón, fussili y espagueti, con las siguientes instrucciones:

1) Hervir el agua en una cacerola

2) Arrojar la pasta en el agua hirviendo

3) Dejar hervir el agua nuevamente, luego de la introducción de la pasta

4) Apagar el fuego

5) Tapar la cacerola para reducir la pérdida de calor

6) Revolver los fideos cada 3 o 4 minutos. 
7) Retirar los fideos luego del tiempo estipulado de cocción.

8) Degustar

Los resultados de este experimento en todas las pastas usadas fueron positivos. No se notaron diferencias apreciables con la pasta cocinada de manera tradicional. Por lo tanto, se determina que el método de cocción compuesto por los pasos 1 a 7 es satisfactorio para cocinar pastas. Experimentos similares han tenido resultados positivos también (Kenji Lopez-Alt, 2010).

Para aplicar este método con pastas largas (espagueti, tallarines, etc.), debe asegurarse que la misma se encuentre sumergida en su totalidad previamente a retirar la fuente de calor. El método no fue evaluado en pastas con relleno, como ravioles o capeletinis.

Se podría cuestionar de este método que las unidades individuales de pastas se "pegarían" al estar en contacto y reposando en el fondo de la cacerola, lo cual no ocurriría en el método tradicional de cocción pues el agua en ebullición mantiene la pasta en movimiento. Sin embargo, el motivo por el cual la pasta se "pega" se debe a que, al comienzo de la cocción, la misma absorbe agua y almidón es liberado a la superficie y se comporta como una especie de pegamento. Pero luego de un tiempo, el almidón de la superficie es lavado por el agua hasta que es completamente removido. Esta es la razón por la cual algunas instrucciones sugieren revolver durante el primer minuto de cocción. Para estimular la remoción del almidón de la superficie, se recomienda aplicar el paso 6 cada 3 o 4 minutos.

Sin embargo, la calidad de cocción de las pastas es una medida cualitativa. Por lo tanto, para robustecer estos resultados se buscó una forma cuantitativa para demostrar que los fideos pueden cocinarse a fuego apagado sin modificar considerablemente el proceso de cocción. La forma seleccionada fue calcular la disminución de temperatura del agua desde un tiempo inicial $\mathrm{t}_{\mathrm{o}}=0$ minutos a una temperatura inicial de $\mathrm{T}=100{ }^{\circ} \mathrm{C}$ hasta una en tiempo final $t_{f}=10$ minutos y una temperatura final $T_{f}$

En la siguiente sección se calculará cuanto gas y cuanta energía se ahorraría en Argentina de aplicarse esta nueva técnica de cocción de pastas.

\section{Segunda parte: Descenso de temperatura de agua en ebullición sin fuente de calor}

Una cacerola con agua caliente a la cual se le ha retirado la fuente de calor pierde energía de tres formas: evaporación de agua, convección natural, y radiación por las paredes de la misma, al ser la temperatura del aire menor a la temperatura de la cacerola. La suma de estas formas de perdida de energía se utilizara para calcular la disminución de la temperatura del agua con la siguiente ecuación:

$$
\Delta T=\frac{\left(Q_{(\mathrm{CN}, \mathrm{R})}+Q_{\mathrm{EV}}\right)}{\mathrm{m} \cdot \mathrm{C}}
$$


Donde $\mathrm{Q}_{(\mathrm{CN}, \mathrm{R})}$ y $\mathrm{Q}_{\mathrm{EV}}$ representan la pérdida de calor por convección natural y radiación en conjunto, $\mathrm{y}$ por evaporación, respectivamente, $\mathrm{m}$ es la masa de agua y $\mathrm{C}$ es la capacidad calorífica del agua.

Se asumirá en este análisis, como condición típica de cocción, $0,5 \mathrm{~kg}$ de pasta (el contenido de 1 paquete) y 5 litros de agua (lo recomendado para cocinar 0,5 $\mathrm{kg}$ de pasta).

\section{Pérdida de Calor por Convección Natural y Radiación}

Las cacerolas usadas para cocinar pasta suelen estar construidas de acero inoxidable y no poseen aislamiento. Por lo tanto, se puede considerar que una cacerola tapada perderá calor al ambiente de manera similar a un tanque de acero inoxidable, dado que la relación entre el radio y la altura son muy similares. Un tanque de acero inoxidable sin aislación a $100{ }^{\circ} \mathrm{C}$ y con una temperatura ambiente de $21,1^{\circ} \mathrm{C}$ posee un factor de pérdida de calor $(\mathrm{F})$ de aproximadamente $1.000 \mathrm{Wh} /$ $\mathrm{m}^{2} / \mathrm{h}\left(3.600 \mathrm{~kJ} / \mathrm{m}^{2} / \mathrm{h}\right)($ Comisión Nacional para el Ahorro de Energía, Gobierno de México, 2007). La perdida de calor por convección es entonces:

$$
\mathrm{Q}_{\mathrm{CN}}=\mathrm{F} \cdot \mathrm{A} \cdot \mathrm{t}
$$

Donde $A$ es el área de la cacerola en contacto con el agua, más el área de la tapa; y $\mathrm{t}$ es el tiempo. Una cacerola típica para cocinar $0,5 \mathrm{~kg}$ de pasta tiene un diámetro de $25 \mathrm{~cm}$. Si se usan 5 litros de agua, la altura del agua será de 10,2 cm. Por lo tanto A será igual a $0,18 \mathrm{~m} 2$. Durante 10 minutos $(0,167 \mathrm{~h})$, la pérdida de calor por convección será:

$$
\mathrm{Q}_{\mathrm{CN}}=3.600 \mathrm{~kJ} /\left(\mathrm{m}^{2} \cdot \mathrm{h}\right) \cdot 0,18 \mathrm{~m}^{2} \cdot 0,167 \mathrm{~h}=107 \mathrm{~kJ}
$$

Este valor es ligeramente sobrestimado, pues a medida que disminuye la temperatura del agua, el factor de pérdida de calor, F, disminuye también, pues la diferencia entre la temperatura del agua y la del ambiente se reduce. Sin embargo, se consideró válido para propósitos estimativos. Considerando una densidad del agua de $1 \mathrm{~kg} / \mathrm{l}$ y una capacidad calorífica del agua de $4,18 \mathrm{~kJ} / \mathrm{kg} /{ }^{\circ} \mathrm{C}$, la disminución de temperatura por convección natural y radiación será:

$$
\Delta \mathrm{T}_{\mathrm{CN}}=\frac{107 \mathrm{~kJ}}{4,18 \mathrm{~kJ} /\left(\mathrm{kg} \cdot{ }^{\circ} \mathrm{C}\right) \cdot 5 \mathrm{~kg}}=5,1^{\circ} \mathrm{C}
$$




\section{Pérdida de Calor por Evaporación}

El calor perdido por evaporación en un tiempo determinado equivale a:

$$
\mathrm{Q}_{\mathrm{EV}}=\mathrm{m}_{\mathrm{EV}}^{\cdot} \cdot \mathrm{t} \cdot \mathrm{H}_{\mathrm{EV}}=\mathrm{m}_{\mathrm{EV}} \cdot \mathrm{H}_{\mathrm{EV}}
$$

Donde $m_{E V}$ corresponde a la tasa de evaporación de agua y $H_{E V}$ es el calor de vaporización del agua.

La fórmula utilizada para calcular la tasa de evaporación es (Schulte, 2008):

$$
\mathrm{m}_{\mathrm{EV}}^{\cdot}=\frac{(201+0.88 \cdot \mathrm{v}) \cdot\left(\mathrm{P}_{\mathrm{A}, \mathrm{S}}-\mathrm{P}_{\mathrm{A}, \mathrm{P}}\right) \cdot \mathrm{A}^{\prime}}{\mathrm{H}_{\mathrm{EV}}}
$$

Donde $m_{E V}$ está dado en $\mathrm{lb} / \mathrm{hr}, v$ es la velocidad del aire en $\mathrm{ft} / \mathrm{min}, P_{A, S}$ es la presión de saturación a la temperatura del agua en milímetros de mercurio $(\mathrm{mmHg}), P_{A, P}$ es la presión parcial del agua en la atmósfera en $\mathrm{mmHg}, A$ ' es el área de exposición del agua al aire en $\mathrm{ft}^{2}$ y $H_{E V}$ es el calor latente del agua en BTU/lb. Los modelos para el cálculo de tasa de evaporación de agua basados en gradiente de concentración o presión de agua, suponen una capa límite inmediatamente superior a la superficie, que está saturada en vapor de agua a la temperatura del agua, y que transfiere masa al aire del ambiente, que posee una presión parcial de agua menor a la de la capa límite. En una cacerola tapada, se asumirá que el espacio entre la superficie de agua y la tapa está saturado en vapor de agua, por lo que cumplirá la función de capa límite. El agua escapará al aire del ambiente por el espacio que la tapa le permita. Asumiendo un espacio anular entre la tapa y el borde de la cacerola de $1 \mathrm{~mm}, \mathrm{o}$ un orificio en la tapa de $3.2 \mathrm{~cm}$ de diámetro, el área $A$ ' será un 1,6\% del área de la tapa, lo cual corresponde a:

$$
\mathrm{A}^{\prime}=\pi \cdot(0,125 \mathrm{~m})^{2} \cdot 0,016=0,00078 \mathrm{~m}^{2}
$$

Se asumirá que la velocidad del aire es igual a cero, al estar una cocina en un espacio cerrado. A $100{ }^{\circ} \mathrm{C}$, la presión de saturación del agua corresponde a la presión atmosférica es igual a $760 \mathrm{mmHg}$. En la ciudad de Buenos Aires, la presión relativa promedio es 72,5\% (Servicio Meteorológico Nacional de la República Argentina, 2013), y a $20^{\circ} \mathrm{C}$, la presión de saturación de agua es aproximadamente $17,5 \mathrm{mmHg}$. Por lo tanto, la presión parcial promedio del agua en la atmósfera de Buenos Aires será:

$$
\mathrm{P}_{\mathrm{A}, \mathrm{P}}=0,725 \cdot 17,5 \mathrm{mmHg}=12,7 \mathrm{mmHg}
$$


A $100{ }^{\circ} \mathrm{C}$, el calor latente del agua es $972 \mathrm{BTU} / \mathrm{lb}$. Finalmente, utilizando la ecuación 1, la masa de agua evaporada en 10 minutos será $0,098 \mathrm{~kg}$. Lo que corresponde a una disminución de la temperatura de:

$$
\Delta \mathrm{T}_{\mathrm{EV}}=\frac{\mathrm{m}_{\mathrm{EV}} \cdot \mathrm{H}_{\mathrm{EV}}}{\mathrm{m} \cdot \mathrm{C}}=\frac{0,098 \mathrm{~kg} \cdot 2.260 \mathrm{~kJ} / \mathrm{kg}}{5 \mathrm{~kg} \cdot 4,18 \mathrm{~kJ} /{ }^{\circ} \mathrm{C} / \mathrm{kg}}=10.6{ }^{\circ} \mathrm{C}
$$

Vale la pena notar que esta disminución de temperatura está sobrestimada pues:

- la presencia de solutos en el agua (ya sea provenientes de la pasta o por la sal que suele echársele al agua) disminuye la evaporación del agua, y

- se asumió que la presión de saturación del agua se mantiene constante a lo largo de los 10 minutos, cuando en realidad disminuye al disminuir la temperatura del agua.

En las referencias consultadas, se encontró también un factor de pérdida de calor por la superficie del agua en tanques abiertos equivalente a $18.500 \mathrm{~W} / \mathrm{m} 2$ (Energy Consumption of Tanks and Vats, 2013). Utilizando este factor para el área A', la disminución de temperatura sería de $0,4{ }^{\circ} \mathrm{C}$. Sin embargo, para ser conservadores, se considerará para el análisis final el primer valor calculado.

La disminución de temperatura debido a la pérdida de calor por convección natural, radiación y evaporación es de $5,1+10,6=15,7^{\circ} \mathrm{C}$. Se concluye, por lo tanto, que la temperatura del agua en una cacerola tapada luego de haberse dejado enfriar por 10 minutos con una temperatura inicial de $100^{\circ} \mathrm{C}$ en una cocina será $100-15.7=84.3$ ${ }^{\circ} \mathrm{C}$. Esta temperatura es mayor a la máxima temperatura de gelatinización encontrada en la bibliografía de $83{ }^{\circ} \mathrm{C}$, aun tomando medidas conservadoras.

\section{Tercera parte: Potencial ahorro de gas y energía en argentina}

En 2011 se consumieron en Argentina 321.225 toneladas de pasta (Organización Internacional de la Pasta, 2012). Suponiendo que cada vez que se consume pasta, se cocina 1 paquete de $500 \mathrm{~g}$ para alimentar a 4 o 5 personas, la cantidad de veces que se cocina pasta por año será:

$$
\frac{321.225 .000 \mathrm{~kg} / \mathrm{año}}{0.5 \mathrm{~kg} / \mathrm{vez}}=642.450 .000 \text { veces al año }
$$

En el nuevo método propuesto, el fuego se apaga al volver a hervir el agua luego de arrojar la pasta. En el método convencional, la hornalla se mantiene prendida durante el tiempo de cocción promedio de 7 minutos. Si se asume 
conservadoramente que el agua vuelve a hervir en 1 minuto luego de arrojar la pasta, entonces el tiempo durante el cual se ahorra gas será 6 minutos. Por lo tanto, el tiempo de ahorro anual será:

$$
\frac{642.450 .000 \mathrm{vez} / \mathrm{año} \cdot 6 \mathrm{~min} / \mathrm{vez}}{60 \mathrm{~min} / \mathrm{hora}}=64.245 .000 \text { horas } / \text { año }
$$

Una hornalla mediana consume en promedio $1.200 \mathrm{kcal} /$ hora (Romero, 2011), y el poder calorífico del gas natural es $9.300 \mathrm{kcal} / \mathrm{Nm} 3$. Si se utilizara el nuevo método para cocinar pastas, el ahorro de consumo de gas por año sería:

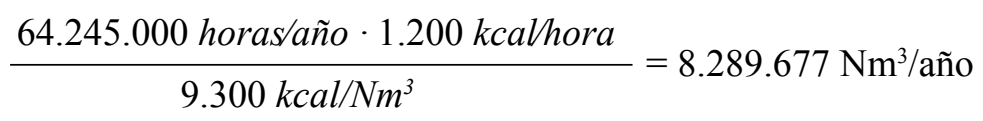

lo cual representa $0,021 \%$ del consumo de gas en Argentina en 2011 (40.268.735 miles de Nm3 (Instituto Argentino de Petróleo y Gas, 2011)), 0,086 \% del consumo de gas residencial en 2011 (9.594.374 miles de Nm3 (IAPG, 2011)), y el consumo de gas de aproximadamente 7.570 casas, pues el consumo anual de gas de una casa de nivel socioeconómico medio es $1.095 \mathrm{Nm} 3$ ("Para evitar sorpresas, sepa cuanto gas consumen sus artefactos", 2009).

La energía ahorrada sería:

8.289.677 Nm3/año $\cdot 9.300 \mathrm{kcalNm} 3=7.709 \cdot 10^{10} \mathrm{kcaVaño}=323 \mathrm{TJ} / \mathrm{año}$

\section{Conclusiones}

Se ha mostrado un método muy simple de ahorro de gas doméstico. Para afrontar una crisis energética y reducir la dependencia de combustibles fósiles, esta investigación sugiere al Código Alimentario Argentino exigir a las empresas productoras de pasta incluir en la etiqueta de sus productos, instrucciones adicionales para cocinar de una forma más energéticamente eficiente.

Esta investigación considera únicamente hornallas de gas. Si el análisis se realizara en hornallas eléctricas, se cree que el resultado del descenso de temperatura sería aun mas favorable, pues la superficie calefactora de la hornalla eléctrica permanece caliente un tiempo considerable luego de haberse apagado. Sin embargo, el cálculo del ahorro de energía sería diferente, al tratarse de diferentes tipos de energía: química o de combustión en hornallas de gas, y eléctrica en hornallas eléctricas. Esta última posee la ventaja ambiental de poder ser abastecida por energías renovables, como la energía solar o eólica. 
Las investigaciones científicas relacionadas a la cocción de alimentos no son abundantes, a pesar de ser un tema que no le escapa a ningún ser humano. En esta ocasión, se observó científicamente la cocción de pastas para investigar potenciales ahorros de energía. Otros métodos de reducción de energía en la cocción de pastas que podrían analizarse en el futuro incluyen la disminución de la temperatura de gelatinización, la reducción de la masa de agua necesaria para cocer las pastas y la utilización de cacerolas que minimicen la pérdida de calor. 


\section{Referencias}

Comisión Nacional para el Ahorro de Energía, Gobierno de México (2007). Estimación de Perdidas de Energía Térmica. Recuperado de http://www.conae. gob.mx/work/sites/CONAE/resources/LocalContent/3856/2/Estimacion_ energia_termica_1_1.pdf

Energy Consumption of Tanks and Vats (2013). En Steam Engineering Principles and Heat Transfer. Recuperado de http://www.spiraxsarco.com/resources/steamengineering-tutorials/steam-engineering-principles-and-heat-transfer/energyconsumption-of-tanks-and-vats.asp/

Ikegwu, O.J., Okechukwu, P.E., y Ekumankana, E.O. (2010) Physico-Chemical and PastingCharacteristics of Flour and Starch from Achi Brachystegiaeurycoma Seed. Journal of FoodTechnology, 8(2) 58-66.DOI: 10.3923/jftech.2010.58.66

Instituto Argentino del Petróleo y del Gas (2011). Consumo de Gas [Archivo de datos]. Disponible en el sitio Web del IAPG http://www.iapg.org.ar/estadisticasnew/ KenjiLopez-Alt, J., (2010). The Food Lab: A new Way to Cook Pasta? SeriousEats. com. Recuperado de http://www.seriouseats.com/2010/05/how-to-cook-pastasalt-water-boiling-tips-the-food-lab.html

Organización Internacional de la Pasta (2012). IPO Annual Survey on World Pasta Industry. Recuperado de http://www.internationalpasta.org/index.aspx?idsub=7/

Romero, S. (2011). J.T.P. Instalaciones Sanitarias y de Gas. U.T.N. Facultad Regional Santa Fe

Para evitar sorpresas, sepa cuanto gas consumen sus artefactos $(2009,22$ de Mayo) Recuperado de http://algoencomunweb.blogspot.com/2009/05/para-evitarsorpresas-sepa-cuanto-gas.html

Salinas Moreno, Y., Herrera Corredor, J.A, Castillo Merino, J., y Pérez Herrera, P. (2003). Cambios físico-químicos del almidón durante la nixtamalización del maíz en variedades con diferente dureza de grano. Archivos Latinoamericanos de Nutrición, 53(2). Recuperado de http://www.scielo.org.ve/scielo.php?pid=S0004 06222003000200011\&script $=$ sci_arttext/

Schulte S.R. (2008, 1 de Enero). Evaporation Water Loss. PFOnline.com. Recuperado de http://www.pfonline.com/articles/evaporation-water-loss/

Servicio Meterológico Nacional de la República Argentina (2013). Características Climáticas de la Ciudad de Buenos Aires. Informes Estacionales [Archivo de datos]. Disponible en el sitio Web del Servicio Meteorológico Nacional, http://www.smn. 
gov.ar/serviciosclimaticos/?mod=elclima\&id $=10$

Vaclavik, V.A. (2002). Fundamentos de ciencia de los alimentos. Huesca: Editorial Acribia.

Villagra, A.A. (2010). Almidón retrogradado en el tratamiento dietoterápico de la Diabetes Mellitus tipo 2. (Trabajo Final Integrador de Licenciatura en Nutrición). Universidad ISALUD, Buenos Aires, Argentina. Recuperado de http://www. isalud.edu.ar/biblioteca/pdf/tf-villagra.pdf/ 